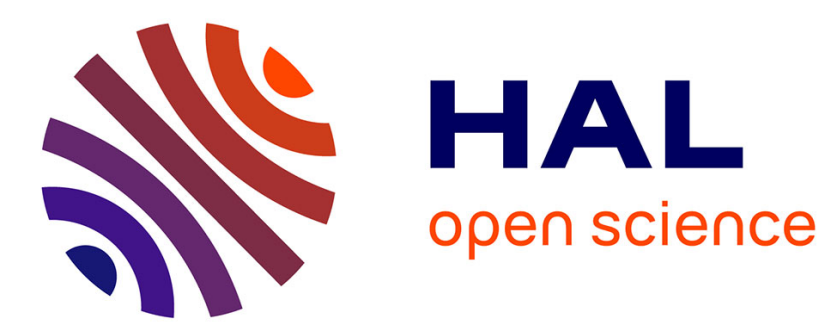

\title{
Characterisation of the volatile fraction of aromatic caramel using heart-cutting multidimensional gas chromatography
}

Laurianne Paravisini, Aurélie Prot, Cécile Gouttefangeas, Cédric Moretton, Henri Nigay, Catherine Dacremont, Elisabeth Guichard

\section{To cite this version:}

Laurianne Paravisini, Aurélie Prot, Cécile Gouttefangeas, Cédric Moretton, Henri Nigay, et al.. Characterisation of the volatile fraction of aromatic caramel using heart-cutting multidimensional gas chromatography. Food Chemistry, 2015, 167, pp.281-289. 10.1016/j.foodchem.2014.06.101 . hal01216254

\section{HAL Id: hal-01216254 \\ https://hal.science/hal-01216254}

Submitted on 15 Oct 2015

HAL is a multi-disciplinary open access archive for the deposit and dissemination of scientific research documents, whether they are published or not. The documents may come from teaching and research institutions in France or abroad, or from public or private research centers.
L'archive ouverte pluridisciplinaire HAL, est destinée au dépôt et à la diffusion de documents scientifiques de niveau recherche, publiés ou non, émanant des établissements d'enseignement et de recherche français ou étrangers, des laboratoires publics ou privés. 


\title{
Characterisation of the volatile fraction of aromatic caramel using heart-cutting multidimensional gas chromatography
}

\author{
Laurianne Paravisini ${ }^{\mathrm{a}, \mathrm{b}, \mathrm{c}, \mathrm{d}}$, Aurélie Prot ${ }^{\mathrm{a}, \mathrm{b}, \mathrm{c}}$, Cécile Gouttefangeas ${ }^{\mathrm{d}}$, Cédric Moretton ${ }^{\mathrm{d}}$, \\ Henri Nigay ${ }^{\mathrm{d}}$, Catherine Dacremont ${ }^{\mathrm{a}, \mathrm{b}, \mathrm{c}, \mathrm{e}}$, Elisabeth Guichard ${ }^{\mathrm{a}, \mathrm{b}, \mathrm{c}, *}$ \\ a INRA, UMR1324 Centre des Sciences du Goût et de l'Alimentation, F-21000 Dijon, France \\ ${ }^{\mathrm{b}}$ CNRS, UMR6265 Centre des Sciences du Goût et de l'Alimentation, F-21000 Dijon, France \\ ${ }^{\mathrm{C}}$ Université de Bourgogne, UMR Centre des Sciences du Goût et de l'Alimentation, F-21000 Dijon, France \\ ${ }^{\mathrm{d}}$ Nigay SA, Z.I. de la Gare, La Féculerie, B.P. 2, F-42110 Feurs, France, \\ ${ }^{\mathrm{e}}$ AgroSup Dijon, 26 rue Petitjean, F-21000 Dijon, France
}

\section{A R T I C L E I N F O}

\section{Article history:}

Received 11 February 2014

Received in revised form 15 May 2014

Accepted 25 June 2014

Available online 3 July 2014

\section{Keywords:}

Heart-cutting multidimensional gas

chromatography (MDGC)

Olfactometry

Mass Spectrometry

Odorant compounds

Caramel

\begin{abstract}
A B S T R A C T
The first aim of our study was to improve characterisation of the volatile fraction of aromatic caramel by applying heart-cutting multidimensional gas chromatography coupled to mass spectrometry and olfactometry (MDGC-MS-O) on targeted odorant fractions. The second aim was to compare the volatile composition of two caramel samples, which differed in terms of their carbohydrate composition and cooking process. MDGC analyses enabled identification of 37 compounds (17 with the addition of pure standard) in the burnt sugar caramel, 20 of which were reported for the first time in caramel. Fifteen compounds were identified as odour-active and described using a range of attributes such as floral, roasted, spicy and almond. Furans, lactones and acids resulting from the thermal breakdown of sugars predominated in the volatile fraction of the burnt sugar caramel, due to the harsher cooking conditions. Finally, these results have enabled a clearer understanding of aromatic caramel as well as the identification of new compounds which might make an important contribution to its aroma.
\end{abstract}

(c) 2014 Published by Elsevier Ltd.

\section{Introduction}

Aromatic caramel results from a non-enzymatic browning reaction that involves the dehydration of sugars during heat treatment under specific conditions, either dry or in a concentrated solution, either alone or with additives. The non-volatile fraction of caramel accounts for $90-95 \%$ of the mass and is composed of high molecular weight compounds such as oligosaccharides and brown polymers. The remaining $5-10 \%$ of the mass represents the volatile fraction which is made up of hundreds of low molecular weight compounds mainly responsible for the typical caramel odour. Because odour is one of the main factors that contributes to consumer acceptability of food products, the identification of volatile compounds is an important concern for the food industry.

Volatile compounds obtained from the thermal degradation of carbohydrates were studied extensively in the 1960s (Fagerson, 1969) but surprisingly, only a few more recent studies have dealt with caramel itself (Cottier, Descotes, Neyret, \& Nigay, 1989;

* Corresponding author. Address: INRA, UMR CSGA, 17 Rue Sully, 21065 Dijon Cedex, France. Tel.: +33 380693277 .

E-mail address: elisabeth.guichard@dijon.inra.fr (E. Guichard).
Goretti, Liberti, \& Dipalo, 1980; Pons, Garrault, Jaubert, Morel, \& Fenyo, 1991). In 2012, the composition of the volatile odorant fraction of four aromatic caramels was investigated using gas chromatography coupled either to olfactometry (GC-O) or mass spectrometry (GC-MS) (Paravisini et al., 2012). Seventy-six zones on the chromatograms appeared to be odour-active according to the GC-O study. Unfortunately, only 49 odorant compounds could be identified by GC-MS analysis, due to the complexity of the caramel volatile fraction which cannot be solved with one-dimensional GC analysis. The number of compounds was too high for a single column peak capacity so the separation was highly compromised. Moreover, the presence of numerous oxygenated heterocyclic compounds such as furans and furanones exhibiting similar interactions with the column stationary phase, led to numerous co-elutions, so that the perceived odour could not be related to a single identified compound.

Multidimensional gas chromatography (MDGC) could be an appropriate technique to overcome identification issues in complex food samples such as caramel. MDGC enables the selection of a targeted fraction on the first dimension. The fraction is then re-separated on a second column that exhibits a different stationary phase. Another advantage of this technique is the use of 
cryo-trapping between the two dimensions that can enhance sensitivity by focusing analytes prior to the second separation (Tranchida, Sciarrone, Dugo, \& Mondello, 2012).

During the past ten years, the MDGC technique has been applied successfully to the aroma characterisation of complex samples such as alcoholic beverages (Mac Namara, Howell, Huang, \& Robbat, 2007), coffee (Miyazato, Nakamura, Hashimoto, \& Hayashi, 2013) and fruits (Darriet et al., 2002). However, to the best of our knowledge, the application of heart-cutting MDGC to the volatile fraction of aromatic caramel has not yet been reported.

The principal purpose of this study was therefore to investigate the volatile composition of caramel by applying MDGC to targeted fractions according to the results of a previous GC/O study. As odour properties are closely related to the cooking properties of caramel, this study also focused on two samples which differed to a considerable extent in terms of their carbohydrate composition and cooking process.

\section{Materials and methods}

\subsection{Samples and chemicals}

Caramel samples were supplied by Nigay SAS (Feurs, France). Caramel A was a standard blond aromatic caramel prepared from sucrose. Caramel B was a burnt sugar type caramel prepared from a mixture of sucrose and glucose under harsher cooking conditions.

The analytical grade ( $>99 \%$ purity) dichloromethane used for the extraction procedure was purchased from Carlo-Erba (Val de Reuil, France) and was re-distilled prior use to ensure the highest possible purity. The chemical standards used to validate identification were purchased from Sigma-Aldrich (Saint Quentin-Fallavier, France).

\subsection{Extraction of volatile compounds}

Caramel samples $(20 \mathrm{~g})$ were mixed with $100 \mathrm{ml}$ deionised water (Milli- $\mathrm{Q}^{\circledR}$, Bedford, MA, USA). Two hundred microlitres of

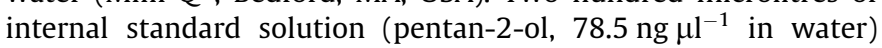
were added to check the repeatability of the extraction procedure. This mixture was introduced into the SAFE (Solvent Assisted Flavour Evaporation) apparatus (Engel, Bahr, \& Schieberle, 1999), and vacuum distillation ( $10^{-3}$ mbar) was performed for two hours. The thermostat of the head and legs of the apparatus was fixed at $35^{\circ} \mathrm{C}$. The same temperature was used to heat the distillation vessel $(500 \mathrm{ml})$ by means of a water bath. The aqueous distillate was then extracted with dichloromethane $(1 \times 20 \mathrm{ml}$ then $2 \times 10 \mathrm{ml})$. The organic phase was collected, filtered through glass wool and dried over anhydrous sodium sulphate. The extract was concentrated to $400 \mu$ l with a Kuderna-Danish apparatus in a $70^{\circ} \mathrm{C}$ water bath, and then to $50 \mu$ under a nitrogen stream. Samples were stored at $-20^{\circ} \mathrm{C}$ prior to chromatographic analysis.

The caramel samples were extracted in triplicate and their repeatability was checked by GC-MS analyses prior to the MDGC experiments. After validation, the three extracts were pooled.

\subsection{Multidimensional Gas Chromatography-Mass Spectrometry- Olfactometry (MDGC-MS-O)}

The separations were performed on a MDGC system equipped with two independent gas chromatographs. Columns were interconnected by means of a Gerstel multi-column switching device (MCS, Gerstel, Germany).

The ${ }^{1} \mathrm{D}$ separation device was a HP 6890 chromatograph (Agilent Technologies, Santa Clara, USA) equipped with a Flame Ionisation Detector (FID) system kept at $250^{\circ} \mathrm{C}$ and a polar DB-Wax fused silica column $(30 \mathrm{~m}$ length $\times 0.32 \mathrm{~mm}$ i.d., $0.5 \mu \mathrm{m}$ film thickness, Agilent J\&W, USA). Sample injections of $2 \mu$ l were made using a 7683 Series autosampler (Agilent Technologies, Santa Clara, USA). Injections were carried out in a Split/Splitless injector kept at $250{ }^{\circ} \mathrm{C}$. The split valve was opened for $30 \mathrm{~s}$ after the injection, with a purge flow of $44 \mathrm{ml} \mathrm{min}{ }^{-1}$. Helium was used as the carrier gas at a constant flow of $1.5 \mathrm{ml} \mathrm{min}^{-1}$. The ${ }^{1} \mathrm{D}$ GC oven temperature started at $40{ }^{\circ} \mathrm{C}$ and was then raised at $3{ }^{\circ} \mathrm{C} \mathrm{min}{ }^{-1}$ up to $240{ }^{\circ} \mathrm{C}$ and kept isothermal for $10 \mathrm{~min}$. The ${ }^{1} \mathrm{D}$ column outlet was connected to the ${ }^{2} \mathrm{D}$ system by means of the MCS switching device. A deactivated fused silica column transferred $10 \%$ split from the MCS device to the FID in order to monitor the eluent from the ${ }^{1} \mathrm{D}$ column. Additional permanent inlet and outlet capillary lines connected the MCS device to a mass flow controller and a pressure regulator module (EPC) which provided a constant inlet flow of $10 \mathrm{ml} \mathrm{min}{ }^{-1}$ through the MCS device, thus allowing the heart-cut procedure. This counter-current flow was switched off during the transfer of a selected heart-cut in the ${ }^{2} \mathrm{D}$ system. Heart-cut fractions were trapped in the first $50 \mathrm{~cm}$ of the ${ }^{2} \mathrm{D}$ column across a cryo trap system (CTS, Gerstel) and then cooled at $-150^{\circ} \mathrm{C}$ with liquid nitrogen. After each heart-cut, the current flow was switched on and the CTS was heated to $250{ }^{\circ} \mathrm{C}$ at a rate of $20^{\circ} \mathrm{C} \mathrm{s}^{-1}$. The EPC module ensured programmed pressure at the head of the ${ }^{2} \mathrm{D}$ column in order to maintain a constant flow of $1.5 \mathrm{ml} \mathrm{min}^{-1}$.

The ${ }^{2}$ D system was an HP 7890A chromatograph (Agilent Technologies, Santa Clara, USA) equipped with a non-polar DB-5MS fused silica column $(30 \mathrm{~m}$ length $\times 0.32 \mathrm{~mm}$ i.d., $0.5 \mu \mathrm{m}$ film thickness, Agilent J\&W, USA). The ${ }^{1} \mathrm{D}$ GC oven temperature started at $40{ }^{\circ} \mathrm{C}$, and was then increased to $240{ }^{\circ} \mathrm{C}$ at a rate of $4{ }^{\circ} \mathrm{C} \mathrm{min}{ }^{-1}$. The eluent of the ${ }^{2} \mathrm{D}$ column was split $2: 1$ between an olfactometric detection port (ODP3, Gerstel, Germany) and a 5975C mass spectrometric detector (Agilent Technologies, Santa Clara, USA). The ODP transfer line was kept at $250^{\circ} \mathrm{C}$. An additional humidified air flow was added to the ODP for the comfort of the panellists. Indeed, two experienced panellists who had participated in the previous GC-O analyses were asked to give a qualitative description when an odorant was perceived. The MS conditions were: transfer line at $150{ }^{\circ} \mathrm{C}$, ion source at $250{ }^{\circ} \mathrm{C}$ and EI voltage at $70 \mathrm{eV}$. Data were recorded in full scan mode $(\mathrm{m} / \mathrm{z}$ range: $29-350$ amu).

\subsection{Selection of the heart-cutting fractions}

Based on a previous GC-O study performed on the same caramel samples (Paravisini et al., 2012), relevant odorant zones for which no compound had been identified because of co-elution or a poor FID signal were selected. The 12 heart-cutting fractions selected, their corresponding odour descriptions and their detection frequencies are shown in Table 1. For each fraction, the heart-cutting times (start and end) were based on the retention indexes (RI) of the GC-O study on the same DB-Wax fused silica column.

\subsection{Identification of compounds}

Compounds were identified on the basis of their RI on the DBWax and DB5MS columns, their mass spectra and the injection of pure standard when available. A series of n-alkanes $\left(C_{10}-C_{30}\right)$ was analysed to establish RI values using the Van Den Dool and Kratz formula (Van den Dool \& Kratz, 1963), which were compared with data from the literature. The mass spectra were compared with those from three databases: NIST 2.0, WILEY 138 and INRAMASS (internal database developed using standard compounds). 
Table 1

Summary of the targeted fractions of the ${ }^{1} \mathrm{D}$ chromatogram according to the previous GC/O study for caramel samples A and B.

\begin{tabular}{|c|c|c|c|c|c|c|}
\hline \multirow{2}{*}{$\begin{array}{l}\text { Heart-cut } \\
\text { No }\end{array}$} & \multicolumn{2}{|l|}{$\mathrm{RI}^{\mathrm{a}}$} & \multirow[t]{2}{*}{ Compound identified by GC/MS } & \multirow[t]{2}{*}{ Odour description GC/O } & \multicolumn{2}{|c|}{ Detection frequency $(\%)^{\mathrm{b}}$} \\
\hline & Start & End & & & Caramel A & Caramel B \\
\hline 1 & 1290 & 1315 & Octanal + 3-hydroxybutan-2-one & Citrus floral mushroom & 39 & 17 \\
\hline 2 & 1517 & 1541 & Benzaldehyde +3 -methylcyclopent-2-en-1-one & Caramel roasted green & 0 & 44 \\
\hline 3 & 1573 & 1591 & 5-Methyl-2-furfural + 2-propionylfuran & Almond floral & 0 & 83 \\
\hline \multirow[t]{2}{*}{4} & 1645 & 1682 & Acetophenone $+2,5$-dihydro-3,5-dimethylfuran-2-one & Coffee roasted & 17 & 6 \\
\hline & & & 2-Methylbutanoic acid + 3-methylbutanoic acid & Cheese & 44 & 78 \\
\hline 5 & 1698 & 1714 & ni & Green roasted hazelnut & 61 & 78 \\
\hline 6 & 1729 & 1750 & Pentanoic acid + cyclohex-2-en-1,4-dione* & Hazelnut chemical cheese & 28 & 39 \\
\hline \multirow[t]{3}{*}{7} & 1830 & 1876 & ni & Roasted & 44 & 72 \\
\hline & & & ni & Caramel lactone & 78 & 39 \\
\hline & & & ni & Chemical pharmaceutical & 0 & 67 \\
\hline 8 & 1905 & 1929 & nd & Lactone & 22 & 39 \\
\hline 9 & 2070 & 2082 & nd & Lactone floral & 33 & 0 \\
\hline \multirow[t]{2}{*}{10} & 2158 & 2192 & nd & Floral lactone spicy & 72 & 61 \\
\hline & & & nd & Floral lactone roasted & 11 & 39 \\
\hline 11 & 2486 & 2512 & 5-(Hydroxymethyl)-2-furfural + dodecanoic acid & Animal sharp & 22 & 28 \\
\hline \multirow[t]{3}{*}{12} & 2543 & 2608 & nd & Chocolate vanilla & 44 & 61 \\
\hline & & & nd & Caramel & 50 & 17 \\
\hline & & & nd & Tobacco & 11 & 11 \\
\hline
\end{tabular}

ni: non identified.

nd: non detected.

a RI on DB-WAX column ( $30 \mathrm{~m}$ length $\times 0.32 \mathrm{~mm}$ i.d., $0.5 \mu \mathrm{m}$ film thickness).

b Detection frequency obtained from GC/O analyses (Paravisini et al., 2012)

${ }^{*}$ Tentatively identified.

\section{Results}

The main objectives of this study were to investigate the composition of caramel by applying MDGC to targeted fractions and to identify volatile compounds by means of MS detection. In addition, in some cases, sensory detection by two panellists from the nine who had performed the GC-O analyses provided further insight into the potential odorant activity of the compounds.

Fig. 1 shows the ${ }^{1} \mathrm{D}$ chromatograms of the caramel extracts and the heart cutting times for the 12 selected fractions (Table 1). Six fractions corresponded to a co-elution of compounds (no 1, 2, 3, 4,6 and 11) while the other six fractions corresponded to unsuccessful identifications on the first dimension.

Fraction no 1 was composed of an odorant zone which was defined as citrus/floral on ${ }^{1} \mathrm{D}$ GC-O, immediately followed by an odorant zone defined as mushroom (Table 1). 3-Hydroxybutan-2one and octanal, which co-eluted on the polar phase, were identified separately on the non-polar phase. Our first hypothesis was that octanal might be responsible for the citrus/floral odour of the first targeted zone. However, MDGC analyses did not support
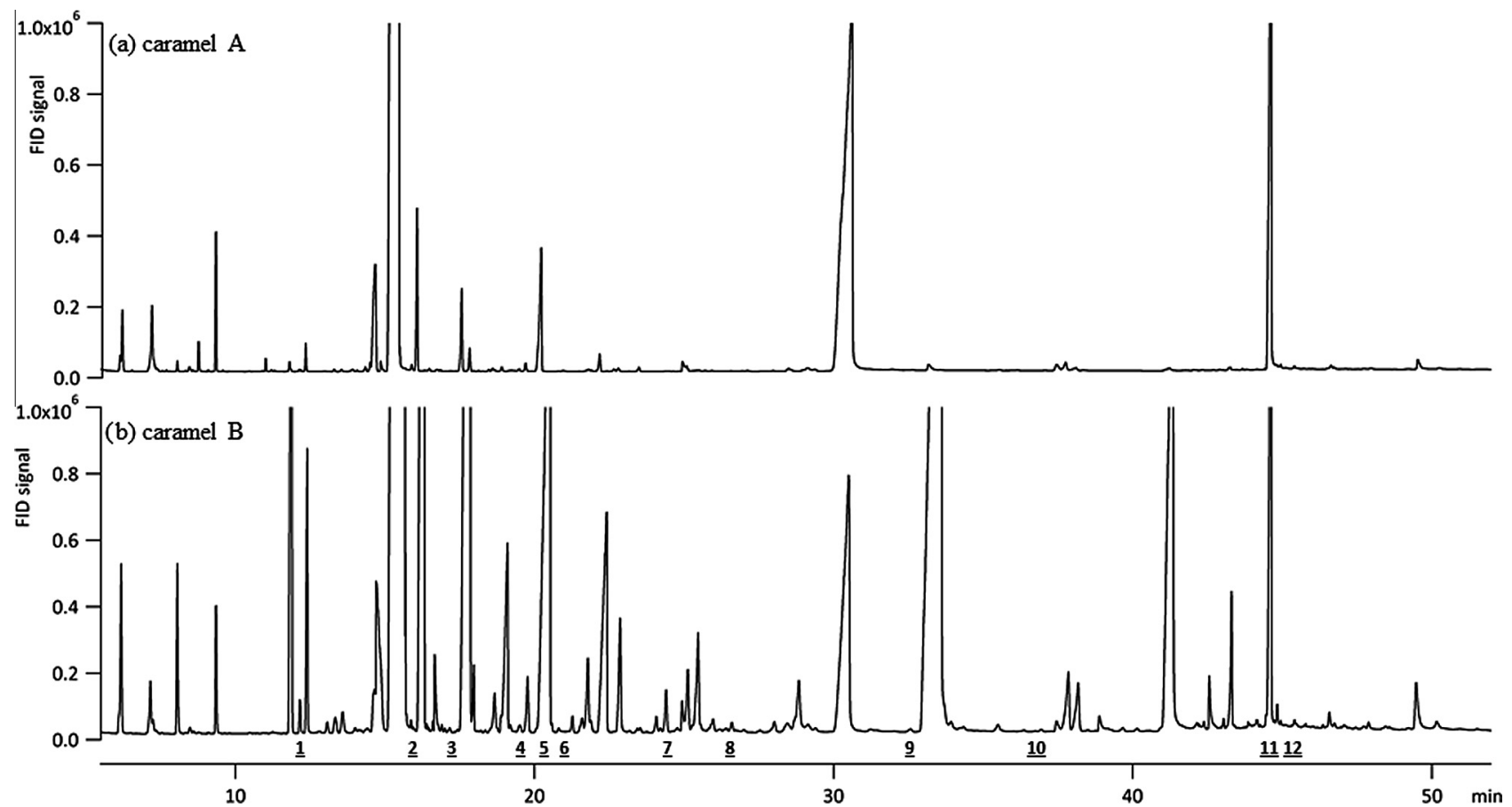

Fig. 1. ${ }^{1}$ D FID chromatogram of the caramel extracts (a: blond caramel A; b: burnt sugar caramel B). 


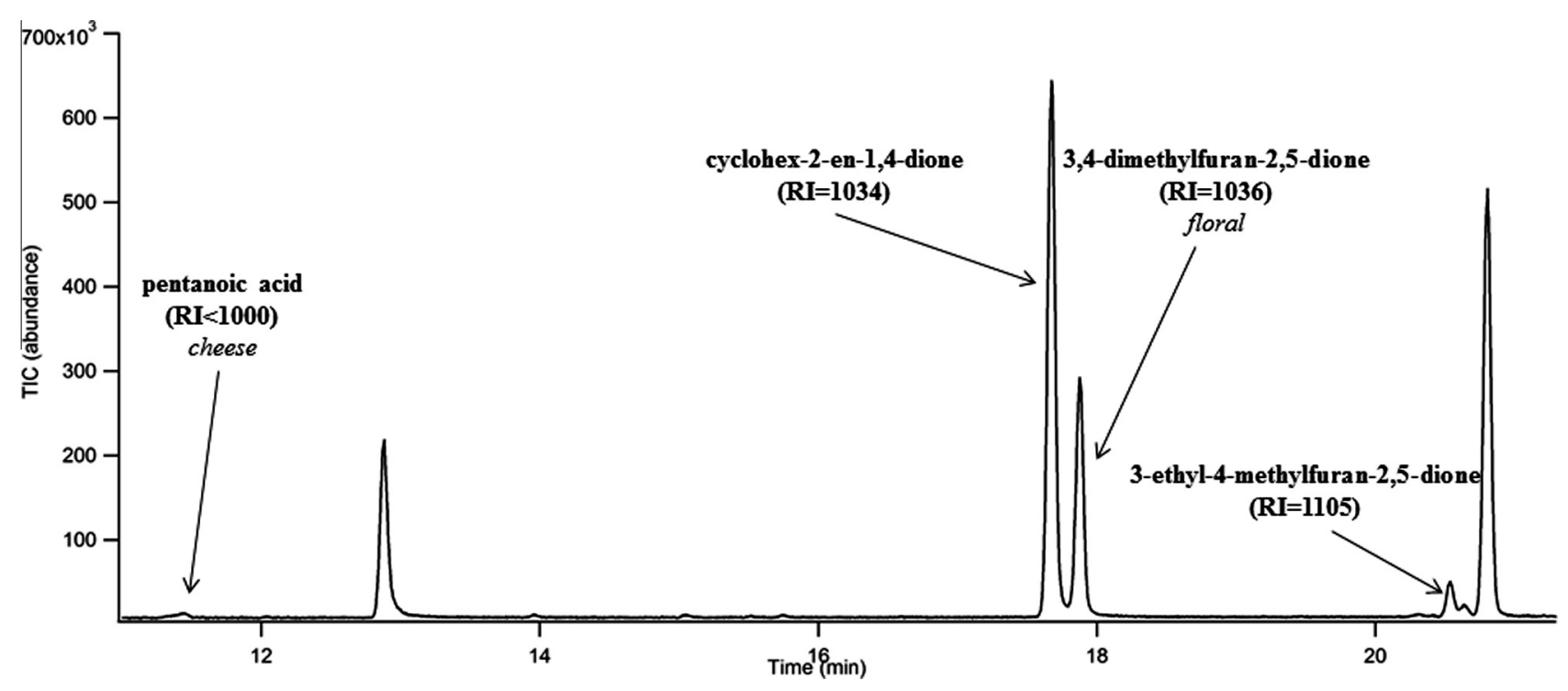

Fig. 2. ${ }^{2} \mathrm{D}$ MS chromatogram of the heart-cut fraction no 6 of the caramel B extract.

this idea as the citrus/floral odour was not reported by the panellists after the ${ }^{2} \mathrm{D}$ separation. It can be assumed that the odour activity of octanal was enhanced while in a mixture with 3-hydroxybutan-2-one but not sufficiently to be detected separately. This type of phenomenon might be due to the existence of perceptive interactions that impact the perception of an odorant compound as a function of context. The mushroom odour of the second targeted zone of fraction no 1 could be clearly associated with the presence of 1-octen-3-one, which was also identified by its mass spectrum.

Finally, another new compound was detected in fraction no 1, but its identification remains uncertain. Its mass spectrum showed a main ion at $m / z 86$ and two fragment ions at $m / z 58$ and 29 (26\% and $21 \%$, respectively, of the abundance of the main ion). This pattern suggests a short-chain carbonyl compound and the fragment ions suggest the loss of a carbonyl group. Such a structure is typical of the intermediate compounds in the formation pathway of furans during the thermal degradation of sugars, such as 3-hydroxy-2butenal, which might be involved in the formation of furan from pentoses (Limacher, Kerler, Davidek, Schmalzried, \& Blank, 2008).

Fraction no 2 was characterised by a caramel/roasted odour associated with a co-elution of benzaldehyde and 3-methylcyclopent-2-en-1-one on ${ }^{1} \mathrm{D}$ GC-O (Table 1). MDGC analyses enabled the separation of 3-methylcyclopent-2-en-1-one and benzaldehyde and the tentative identification of 3-methylfurane-2,5-dione. According to the olfactometric data, the caramel/roasted odour could be attributed to the presence of 3-methylcyclopent-2-en-1one.

In fraction no 3, 5-methyl-2-furfural was identified and described as having a nutty odour note, i.e. almond, vanilla and cinnamon. In addition, 2-propionylfuran and 2-acetyl-5-methylfuran were tentatively identified and the panellists reported a floral note for the latter. Here, MDGC analyses produced results that were consistent with the ${ }^{1} \mathrm{D}$ GC-O findings (Table 1 ) and also enabled the tentative identification of a new compound, 2-acetyl-5methylfuran.

The heart-cutting of fraction no 4 led to the identification of six compounds, four of which were not identified by the ${ }^{1} \mathrm{D}$ analysis. The 2- and 3-methylbutanoic acids detected as a mixture from the first column were separated on the non-polar phase of the second dimension. After this non-polar separation, the cheese odour of the acids (Table 1) was not reported in MDGC analyses. As seen in fraction no 1 with octanal, it can be assumed that the odour activity of 2- and 3-methylbutanoic acids was enhanced while in a mixture and was not sufficient if detected separately. 2,5-Dihydro-3,5-dimethylfuran-2-one was described as having a roasted/ curry odour note, a description in line with the GC-O description of zone 4 in the GC-O analysis (Table 1). Two furans were also detected: 1-(2-furanyl)-butanone, described as having a plastic/ urine/phenolic odour, and 2-methyl-5-propionylfuran.

In fraction no 6 , pentanoic acid was detected at $t_{R}=11.4 \mathrm{~min}$ $(\mathrm{RI}<1000)$ and its cheesy odour was reported (Fig. 2). Cyclohex2-en-1,4-dione, which co-eluted with pentanoic acid on the polar phase, was identified at $t_{R}=17.8 \mathrm{~min}(\mathrm{RI}=1034)$ with no detectable odour under MDGC conditions. This led us to validate the important contribution of pentanoic acid to the odour of this targeted fraction. Two other compounds were also identified by ${ }^{2} \mathrm{D}$ GC-O: 3,4-dimethylfuran-2,5-dione, with a floral odour, and 3ethyl-4-methylfuran-2,5-dione.

The ${ }^{2} \mathrm{D}$ analysis of fraction no 11 allowed the successful separation of 5-hydroxymethylfurfural (5-HMF) co-eluted with dodecanoic acid on the polar phase. This fraction was characterised as having an animal and sharp odour under ${ }^{1} \mathrm{D}$ GC-O analysis (Table 1), which might have been associated with the presence of 5-HMF. This compound was indeed associated with a musty odour during MDGC analyses, whereas dodecanoic acid was not detected at the sniffing port. The divergence between the ${ }^{1} \mathrm{D}$ GC-O and MDGC descriptors might have been due to co-elution with dodecanoic acid, which might enhance or suppress some of the odorant properties of 5-HMF.

Analyses of fractions no 5, 7, 8, 9, 10 and 12 were more challenging because no compound could be identified under ${ }^{1} \mathrm{D}$ analysis. The MDGC analyses allowed us to identify new compounds in these fractions (Table 2), thus highlighting a greater separation capacity.

Fraction no 5 had previously been characterised by green, roasted and nutty odour notes (Table 1 ). The co-occurrence of descriptors belonging to different odour categories, such as green and roasted, suggested a co-elution of several unidentified compounds. Six compounds were separated on the ${ }^{2} \mathrm{D}$ MS chromatogram and three were identified: 3-methyl-2(5H)-furanone, $\gamma$-hexalactone and 1-(3-hydroxy-2-furyl)ethanone, better known as isomaltol. The three other compounds remain unidentified. While the green, roasted and nutty odours found by the ${ }^{1} \mathrm{D} G \mathrm{G} / \mathrm{O}$ 
Table 2

Results of the MDGC/MS/O analyses performed on the caramel samples A and B.

\begin{tabular}{|c|c|c|c|c|c|c|c|c|c|c|}
\hline \multirow[t]{2}{*}{ No } & \multicolumn{2}{|c|}{ RI non-polar } & \multicolumn{2}{|l|}{ RI polar } & \multirow[t]{2}{*}{ No heart-cut } & \multirow[t]{2}{*}{ Compounds $^{4}$} & \multirow[t]{2}{*}{ Odour description } & \multirow[t]{2}{*}{ Caramel A } & \multirow[t]{2}{*}{ Caramel B } & \multirow[t]{2}{*}{ Identification $^{5}$} \\
\hline & Exp. $^{1}$ & Lit. $^{3}$ & Exp. $^{2}$ & Lit. $^{3}$ & & & & & & \\
\hline 1 & $<1000$ & 740 & 1287 & 1297 & 1 & 3-Hydroxybutan-2-one ${ }^{b, d}$ & & $\mathrm{x}$ & $\mathrm{x}$ & MS, RI, std \\
\hline 2 & $<1000$ & & $1290-1315$ & & 1 & ni & & & $\mathrm{x}$ & \\
\hline 3 & $<1000$ & 980 & $1290-1315$ & 1313 & 1 & 1-Octen-3-one & Mushroom & & $\mathrm{x}$ & MS, RI, std \\
\hline 4 & 1010 & 1009 & 1287 & 1297 & 1 & Octanal & & $\mathrm{x}$ & $\mathrm{x}$ & MS, RI, std \\
\hline 5 & $<1000$ & 949 & $1517-1541$ & & 2 & 3-Methyl-2,5-furanedione & & & $\mathrm{x}$ & MS \\
\hline 6 & $<1000$ & 975 & 1532 & 1513 & 2 & 3-Methylcyclopent-2-en-1-one & Caramel roasted & & $\mathrm{x}$ & MS, RI, std \\
\hline 7 & $<1000$ & 964 & 1532 & 1528 & 2 & Benzaldehyde & & $\mathrm{x}$ & $\mathrm{X}$ & MS, RI, std \\
\hline 8 & $<1000$ & 957 & 1578 & 1575 & 3 & 5-Methyl-2-furfural & Almond vanilla cinnamon & $\mathrm{x}$ & $\mathrm{x}$ & MS, RI, std \\
\hline 9 & 1012 & 1005 & 1578 & 1567 & 3 & 2-Propionylfuran ${ }^{\mathrm{a} *}$ & & & $\mathrm{X}$ & MS, RI \\
\hline 10 & 1050 & 985 & $1573-1591$ & & 3 & 2-Acetyl-5-methylfuran & Urine floral & & $\mathrm{x}$ & MS \\
\hline 11 & $<1000$ & 876 & 1679 & 1685 & 4 & 3-Methylbutanoic acid & & & $\mathrm{X}$ & MS, RI, std \\
\hline 12 & $<1000$ & 873 & 1679 & 1685 & 4 & 2-Methylbutanoic acid & & & $\mathrm{x}$ & MS, RI, std \\
\hline 13 & 1004 & & 1660 & 1645 & 4 & 2,5-Dihydro-3,5-dimethylfuran-2-one* & Roasted curry & & $\mathrm{x}$ & MS, RI \\
\hline 14 & 1077 & 1070 & 1660 & 1649 & 4 & Acetophenone & & & $\mathrm{x}$ & MS, RI, std \\
\hline 15 & 1106 & & $1645-1682$ & & 4 & 1-(2-Furanyl)-1-butanone & Plastic urine phenol & & $\mathrm{x}$ & MS \\
\hline 16 & 1104 & 1108 & $1645-1682$ & & 4 & 2-Methyl-5-propionylfuran ${ }^{\mathrm{a} *}$ & & & $\mathrm{x}$ & MS, RI \\
\hline 17 & $<1000$ & 983 & $1698-1714$ & & 5 & 3-Methyl-2(5H)-furanone & & $\mathrm{x}$ & $\mathrm{x}$ & MS \\
\hline 18 & $<1000$ & 989 & $1698-1714$ & & 5 & 1-(3-Hydroxy-2-furyl)ethanone (isomaltol) $)^{a *}$ & Caramel & $\mathrm{x}$ & $\mathrm{x}$ & MS \\
\hline 19 & $<1000$ & & $1698-1714$ & & 5 & ni & & & $\mathrm{x}$ & \\
\hline 20 & 1048 & & $1698-1714$ & & 5 & $\mathrm{ni}$ & & & $\mathrm{x}$ & \\
\hline 21 & 1059 & 1066 & 1702 & 1700 & 5 & $\gamma$-Hexalactone & & & $\mathrm{x}$ & MS, RI, std \\
\hline 22 & 1089 & & $1698-1714$ & & 5 & ni & & & $\mathrm{x}$ & \\
\hline 23 & $<1000$ & 926 & 1745 & 1744 & 6 & Pentanoic acid ${ }^{\mathrm{c}}$ & Cheese sharp & & $\mathrm{x}$ & MS, RI, std \\
\hline 24 & $<1000$ & & $1726-1750$ & & 6 & ni & & & $\mathrm{x}$ & \\
\hline 25 & 1034 & 1024 & 1745 & 1744 & 6 & Cyclohex-2-en-1,4-dione & & & $\mathrm{x}$ & MS, RI \\
\hline 26 & 1036 & & $1726-1750$ & & 6 & 3,4-Dimethylfuran-2,5-dione & Floral & & $\mathrm{x}$ & MS \\
\hline 27 & 1105 & & $1726-1750$ & & 6 & 3-Ethyl-4-methylfuran-2,5-dione & & $\mathrm{x}$ & $\mathrm{x}$ & MS \\
\hline 28 & 1108 & & $1726-1750$ & & 6 & ni & & & $\mathrm{x}$ & \\
\hline 29 & 1000 & 1000 & $1830-1876$ & 1830 & 7 & Hexanoic acid ${ }^{c, b, *}$ & Sharp spicy & $\mathrm{x}$ & $\mathrm{x}$ & MS, RI \\
\hline 30 & 1040 & & $1830-1876$ & & 7 & 5,6-Dihydro-2H-pyran-2-one & & & $\mathrm{x}$ & MS \\
\hline 31 & 1043 & 1030 & $1830-1876$ & 1865 & 7 & Benzyl alcohol & & $\mathrm{x}$ & $\mathrm{x}$ & MS, RI \\
\hline 32 & 1048 & 1036 & $1830-1876$ & & 7 & 4-Methyl-(5H)-furan-2-one & Roasted & $\mathrm{x}$ & $\mathrm{x}$ & MS, RI \\
\hline 33 & 1075 & & $1830-1876$ & & 7 & ni & & $\mathrm{x}$ & $\mathrm{x}$ & \\
\hline 34 & 1084 & & $1830-1876$ & & 7 & ni & & $\mathrm{x}$ & $\mathrm{x}$ & \\
\hline 35 & 1093 & 1090 & $1830-1876$ & 1859 & 7 & Guaiacol $^{*}$ & Pharmaceutical & & $\mathrm{x}$ & MS, RI \\
\hline 36 & 1100 & & $1830-1876$ & & 7 & ni & & $\mathrm{x}$ & $\mathrm{x}$ & \\
\hline 37 & 1108 & & $1830-1876$ & & 7 & 3,4-Dimethyl-2(5H)-furanone ${ }^{*}$ & & $\mathrm{x}$ & $\mathrm{x}$ & MS \\
\hline 38 & 1116 & & $1830-1876$ & & 7 & ni & & $\mathrm{x}$ & $\mathrm{x}$ & \\
\hline 39 & 1120 & & $1830-1876$ & & 7 & $\mathrm{ni}$ & & $\mathrm{x}$ & $\mathrm{x}$ & \\
\hline 40 & 1175 & & $1830-1876$ & & 7 & $\mathrm{ni}$ & & $\mathrm{x}$ & $\mathrm{x}$ & \\
\hline 41 & 1189 & & $1830-1876$ & & 7 & ni & & $\mathrm{x}$ & $\mathrm{x}$ & \\
\hline 42 & 1123 & & 1905-1929 & & 8 & ni & & $\mathrm{x}$ & $\mathrm{x}$ & \\
\hline 43 & 1139 & & $1905-1929$ & & 8 & 2,4-Dimethylcyclopentan-1,3-dione ${ }^{*}$ & & & $\mathrm{x}$ & MS \\
\hline 44 & 1173 & & 1905-1929 & & 8 & ni & & & $\mathrm{x}$ & \\
\hline 45 & 1192 & & 1905-1929 & & 8 & $\mathrm{ni}$ & & $\mathrm{x}$ & $\mathrm{x}$ & \\
\hline 46 & 1226 & & $1905-1929$ & & 8 & ni & & $\mathrm{x}$ & $\mathrm{x}$ & \\
\hline 47 & 1267 & 1266 & 1905-1929 & 1929 & 8 & $\gamma$-Octalactone & & $\mathrm{x}$ & $\mathrm{x}$ & MS, RI, std \\
\hline 48 & 1130 & 1136 & $2066-2082$ & & 9 & 5-Acetyldihydro-(3H)-furan-2-one (solerone) & & $\mathrm{x}$ & $\mathrm{x}$ & MS \\
\hline 49 & 1272 & 1275 & $2158-2192$ & 2200 & 10 & Nonanoic Acid" & Spicy smoked & $\mathrm{x}$ & $\mathrm{X}$ & MS, RI \\
\hline 50 & 1478 & 1468 & $2158-2192$ & 2159 & 10 & $\gamma$-Decalactone & & & $\mathrm{x}$ & MS, RI, std \\
\hline
\end{tabular}




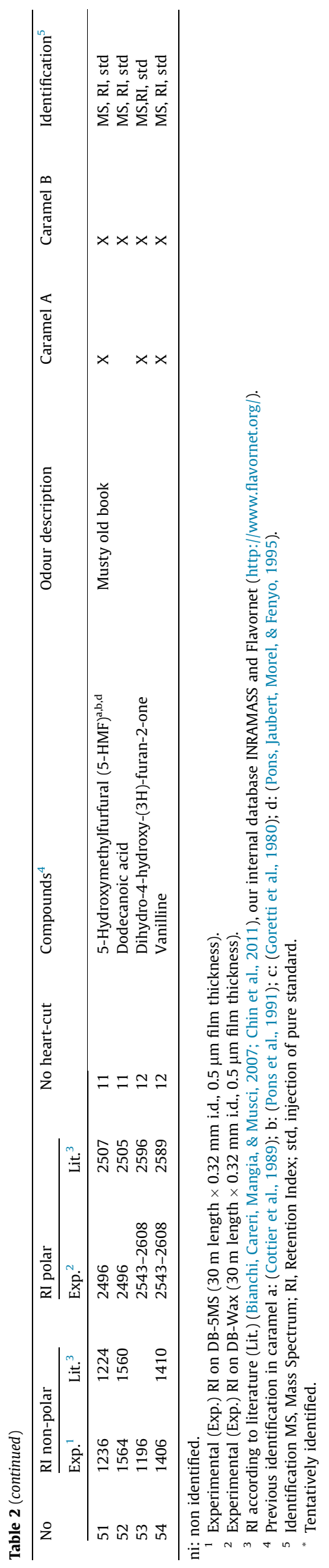

analyses were not reported in MDGC analyses, isomaltol was described with a typical caramel odour. Once again, the divergence between the odorant descriptions might have been linked to the existence of a co-elution on the first dimension. Although isomaltol is well-known for its sweet caramel odour (Pittet, Rittersbacher, \& Muralidhara, 1970), its odorant properties in a mixture with five compounds cannot yet be predicted.

Fraction no 7 was characterised by roasted, caramel/lactone and pharmaceutical odour notes by GC-O analyses (Table 1 ) but no compound could be identified after GC-MS analysis due to several co-elutions which rendered the mass spectra uninterpretable. The ${ }^{2} \mathrm{D}$ MS chromatogram of this heart-cut fraction from caramel B extract is given in Fig. 3. Thirteen compounds were detected, of which seven were identified by MS and three showed odour activity. Hexanoic acid was described as having a sharp and spicy odour. 4-Methyl-(5H)-furan-2-one $(\mathrm{RI}=1048)$ and guaiacol $(\mathrm{RI}=1093)$ were respectively characterised by roasted and pharmaceutical odour notes.

The ${ }^{2} \mathrm{D}$ analysis of fraction no 8 led to the detection of six compounds, two of which were identified: 2,4-dimethylcyclopentan1,3-dione and $\gamma$-octalactone. In fraction no 9 , previously associated with a lactone and floral odour, only 5-acetyldihydro-(3H)-furan2 -one (or solerone) was identified. In fraction no 10 , described as being floral, lactone, spicy and roasted after ${ }^{1} \mathrm{D}$ GC-O analyses, only nonanoic acid was described with a spicy and smoked odour by our panellists following ${ }^{2} \mathrm{D}$ GC-O analyses. $\gamma$-Decalactone was identified at RI $=1478$ in the same fraction but with no detectable odour.

In these fractions no 8, 9 and 10, olfactometric detection did not provide any pertinent information regarding the qualitative properties of the compounds and particularly concerning lactone odours. In this case, the small number of panellists (only two) was probably the cause.

In fraction no 12, described as having chocolate vanilla/caramel/ tobacco odours, two new compounds were identified: dihydro-4hydroxy-(3H)-furan-2-one and vanillin. Neither was detected by our panellists. We can therefore assume that vanillin was the main contributor to the vanilla note, but further analyses are necessary with a larger number of panellists to confirm this finding.

\section{Discussion}

Multidimensional gas chromatography appears to be an efficient way to overcome identification issues in complex food samples such as caramel. In particular, heart-cutting is a suitable approach to investigate the composition of targeted fractions. This study focused on 12 odorant fractions in which compounds potentially making an important contribution to aroma could not be identified by ${ }^{1} \mathrm{D}$ GC-MS analyses. Olfactometric detection at the end of ${ }^{2} \mathrm{D}$ GC was performed by two experienced panellists in order to obtain a qualitative description of the odorants. The results revealed some divergences from the ${ }^{1} \mathrm{D}$ GC-O results, probably due to the smaller number of panellists in the MDGC study. Indeed, one of the main disadvantages of characterisation technique such as heart-cutting, is the large number of analyses required for just one sample. Then, for practical reasons, it is practically impossible to perform a quantitative GC-O study with a dozen panellists. Comprehensive two-dimensional gas chromatography $(\mathrm{GC} \times \mathrm{GC})$ has recently been the subject of intense development and has now become one of the most powerful techniques in terms of resolution (Seeley \& Seeley, 2013). This technique enables the full characterisation of a sample in two orthogonally stationary phases with only one run. However, it also necessitates the use of "fast" detectors such as Time of Flight-MS (TOF-MS) or Flame Ionisation Detector (FID) because of the very high separation speed on the 


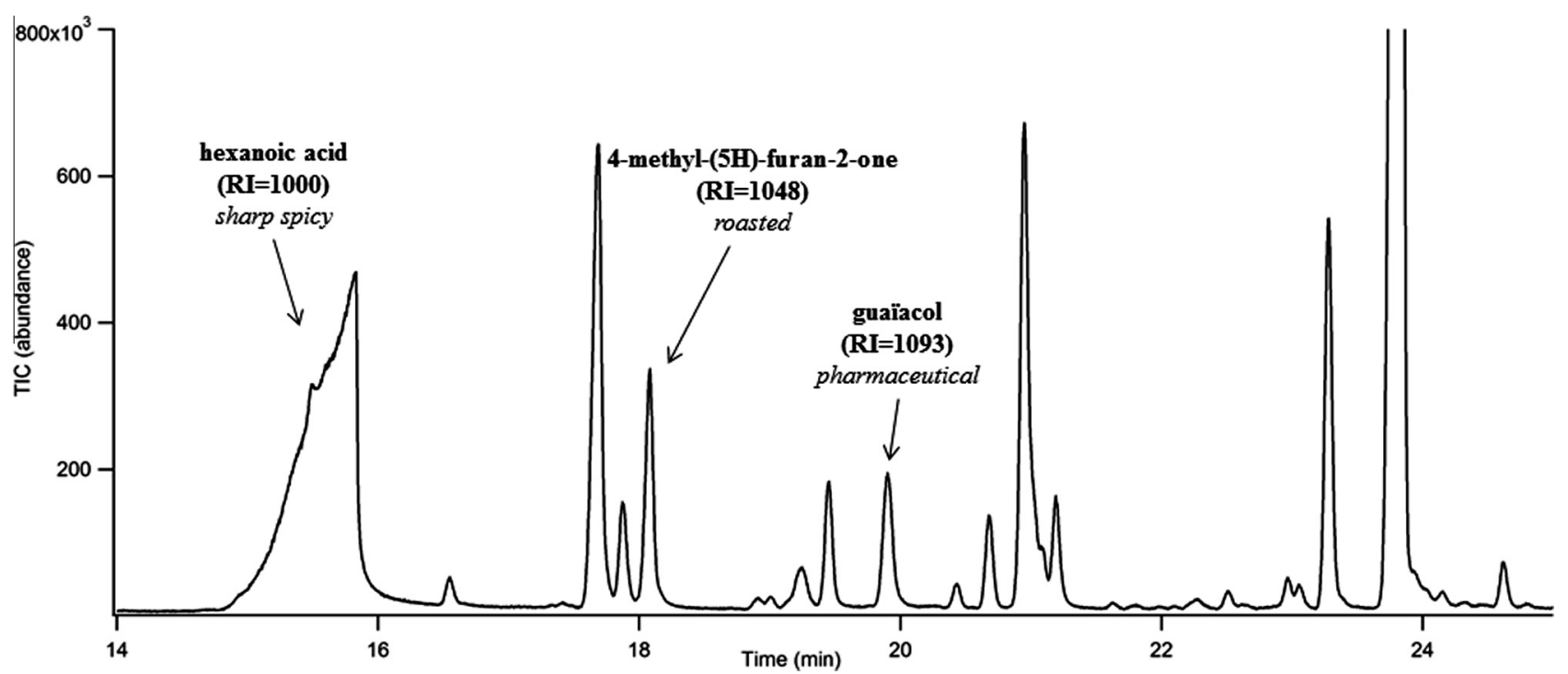

Fig. 3. ${ }^{2} \mathrm{D}$ MS chromatogram of the heart-cut fraction no 7 of the caramel B extract.

second column. Thus combining GC $\times$ GC with olfactometric detection is not possible because of the slow breathing rate of humans.

Another putative explanation for the discrepancy found between odour descriptions concerns the existence of synergistic effects between the co-eluted compounds. For example, according to our previous results, numerous odorant zones were characterised by fruity and lactone descriptors. However, only a few lactones were detected by the nose after ${ }^{2} \mathrm{D}$ GC-O analyses. The odour activity of lactones may be enhanced by the presence of other compounds in co-elution, so that the mixture may be detected but not each compound separately. Miyazawa et al. demonstrated the synergy that occurs between carboxylic acids and maple lactone (or 2-hydroxy-3-methylcyclopent-2-ene-1-one) (Miyazawa, Gallagher, Preti, \& Wise, 2008). The presence of acetic and butyric acids at low concentrations rendered the odour of maple lactone more easily detectable. We can assume that a similar phenomenon occurs when lactones are co-eluted with other compounds after ${ }^{1} \mathrm{D}$ separation.

Nevertheless, the use of MDGC enabled the MS detection of 54 compounds (Table 2) from the heart cutting of only 12 fractions. Seventeen compounds remained unidentified due to non-indexed mass spectra and the presence of numerous isomers hampering their identification. To our knowledge, 20 compounds were reported for the first time in caramel, which thus constituted the added value of this study as a significant contribution to scientific knowledge on caramel. Most of the volatile compounds were furans and furan derivatives, carboxylic acids and lactones. We also counted four aromatic compounds, two ketones and one aldehyde. Furans and furan derivatives are the principal compounds that arise from the thermal transformation of sugars, so most have already been widely reported in caramel. In line with the literature, isomaltol (1-(3-hydroxy-2-furyl)ethanone) was identified and described as having a caramel odour (Pittet et al., 1970). This compound can easily be obtained from the thermal degradation of sucrose in aqueous solution at $120^{\circ} \mathrm{C}$ (Ito, 1977) and has already been reported in caramel (Cottier et al., 1989). However, 2acetyl-5-methylfuran and 1-(2-furanyl)-butanone were reported for the first time in caramel, with urine, floral and phenolic odour notes. Most of these compounds have previously been reported in food products undergoing a thermal treatment, such as coffee (Chin, Eyres, \& Marriott, 2011). Major differences were observed between the two caramel samples in terms of their furan composition. Among the seven furans identified, only three of them: 5-methylfurfural, isomaltol, and 5-HMF, were detected in the blond caramel A. We can assume that their formation was favoured by the cooking process used for caramel B.

Six carboxylic acids were identified in the burnt sugar caramel B: 2- and 3-methylbutanoic, pentanoic, hexanoic, nonanoic and decanoic acids. Some of these (methylbutanoic, pentanoic and hexanoic acids) had previously been reported in caramel (Goretti et al., 1980; Pons et al., 1991), but the presence of nonanoic and decanoic acids is reported here for the first time. Acids are well-known to contribute to food aromas; for example in dairy products, where they directly contribute to the lactic and cheesy odour (Thomsen et al., 2012). The hexanoic acid odour was described as being sharp and spicy. This odorant is known to occur in many food products such as chocolate where it has a pungent odour (Schnermann \& Schieberle, 1997), and in wine where its odour is described as tobacco, floral and toasty (Miranda-Lopez, Libbey, Watson, \& McDaniel, 1992). Recently, it was shown that carboxylic acids constitute one of the main chemical classes identified in the volatile fraction of caramel and are responsible for the pungent odour notes of burnt sugar type caramels (Paravisini et al., 2012). This supports our findings regarding the identification of only two out of six acids in the blond caramel $\mathrm{A}$.

MDGC analysis was useful for the identification of lactones, or furanones, in the caramel extracts. Indeed, thanks to a better resolution, 12 lactones were detected and identified rather than just one after ${ }^{1} \mathrm{D}$ analysis. 2,5-Dihydro-3,5-dimethylfuran-2-one and 4-methyl-(5H)-furan-2-one were associated with roasted notes, and the odour of 3,4-dimethylfuran-2,5-dione was described as floral. The other nine lactones were only identified by MS. The occurrence of solerone has already been reported in wine. However its impact on wine aroma is weak, even at its highest natural concentration (Martin, Etiévant, \& Le Quéré, 1991). Other authors have reported its odour in dried figs as a weak note of dried fruits (Naef, Jaquier, Boschung, \& Lindstroem, 1995). This may explain why this compound was not smelt in our caramel extracts.

Different pathways may explain the formation of lactones, such as the metabolomic pathways of micro-organisms (Feron, Bonnarme, \& Durand, 1996) or the thermal degradation of sugars (Beck, Ledl, Sengl, \& Severin, 1990). Therefore, lactones occur in many food aromas; for example, $\gamma$-lactones are typical compounds of fruity aromas (Guichard, Kustermann, \& Mosandl, 1990). But to 
the best of our knowledge, the 12 lactones we identified are reported here for the first time in aromatic caramel.

The identification of 2-methoxyphenol (guaiacol) and 4hydroxy-3-methylbenzaldehyde (vanillin) was one of the main findings of this study. Indeed, in fractions no 7 and 12 respectively, pharmaceutical and vanilla odour notes were detected during the ${ }^{1}$ D GC-O study (Table 1 ), but no compounds could be identified. The RI on the polar phase of these fractions gave some clues as to the presence of these two compounds but their MS ions were not found. The MDGC analysis enabled the identification of guaiacol with both olfactometry and MS detection. Vanillin was only identified through its mass spectrum. Once again, the contribution of these compounds in caramel has never previously been reported, whereas their occurrence in food products resulting from a non-enzymatic browning reaction has been described several times (Dorfner, Ferge, Kettrup, Zimmermann, \& Yeretzian, 2003). It should be noted that guaiacol was only detected in the burnt sugar caramel, while vanillin was found in both caramels.

Benzaldehyde and 3-methylcyclopent-2-en-1-one were identified in fraction no 2 but only 3-methylcyclopent-2-en-1-one displayed any odour activity. These compounds had both been reported as being obtained by the thermal degradation of sugars (Baltes \& Mevissen, 1988) but their contribution to caramel aroma was still unclear. 3-Methylcyclopent-2-en-1-one was only detected in the burnt sugar type caramel, suggesting its preferential formation under harsher cooking conditions. This compound has previously been reported in Arabica coffee characterised by a roasted note (Sanz, Maetztu, Zapelena, Bello, \& Cid, 2002).

Finally, 1-octen-3-one was identified in the heart-cutting analysis of fraction no 1 from the burnt sugar caramel. Despite the detection of a mushroom odour note in the blond caramel A by ${ }^{1}$ D GC-O analyses (Paravisini et al., 2012), 1-octen-3-one could not be detected by ${ }^{2} \mathrm{D}$ GC-O. As 1 -octen-3-one was detected in sample $B$, this lack of detection could not have been due to the panellists' lack of sensitivity. Even if its occurrence in caramel has not previously been reported, 1-octen-3-one is well-known for its powerful odorant properties because of its very low odour threshold (5 $\mu \mathrm{g} \mathrm{l}^{-1}$ in water (Buttery, Teranishi, Flath, \& Ling, 1990)) and it is present in various food aromas such as cheese (Zehentbauer \& Reineccius, 2002) and vinegar (Acena, Vera, Guasch, Busto, \& Mestres, 2011).

Our results therefore showed that only 27 compounds in the targeted fractions were detected in the blond caramel $\mathrm{A}$, whereas 54 were detected in the burnt sugars $B$. No specific compound could be attributed to caramel $A$, which allows us to theorise that the differences observed were due to a preferential formation of the identified compounds under harsher cooking conditions. Further analyses involving a broad range of caramel samples varying in terms of their initial sugar composition and process now need to be performed in order to validate our findings and help us to better understand their sensory properties.

\section{Conclusion}

The heart-cutting MDGC technique enabled the identification of new compounds, some of which were reported for the first time in caramel and may be important contributors to its aroma.

The predominance of furans, lactones and acids in the burnt sugar caramel showed that their formation is favoured by harsher cooking conditions. These compounds may originate from the thermal breakdown of sugars. They also result from the degradation of brown high molecular weight (HMW) compounds that are found at much higher concentrations in burnt sugar than in blond caramel.

The ${ }^{2} \mathrm{D}$ analyses clearly identified the compounds responsible for the odour of the whole fraction. However, some differences were observed between the ${ }^{2} \mathrm{D}$ and ${ }^{1} \mathrm{D}$ odour descriptions. There may be several explanations for this. First, such a discrepancy could be explained by a lack of sensitivity of the two panellists involved in the ${ }^{2} \mathrm{D}$ study when compared to the ${ }^{1} \mathrm{D}$ study which was carried out with a larger number of panellists, thus compensating for the low sensitivity of some of them. Second, the odour of specific compounds may be enhanced in a mixture with other compounds, whereas they exhibit no odour activity alone. Finally, the odour of a mixture of odorants may differ from that of the individual compounds. These findings could be explained by masking or fusion phenomena resulting from perceptive interactions.

\section{Acknowledgements}

This work received financial support from the Nigay SAS, ANRT (Association Nationale de la Recherche et de la Technologie), the Regional Council for Burgundy and FEDER (European Union). The authors would also like to thank Karine Gourrat and the ChemoSens platform (INRA) for their technical support.

\section{References}

Acena, L., Vera, L., Guasch, J., Busto, O., \& Mestres, M. (2011). Chemical characterization of commercial sherry vinegar aroma by headspace solidphase microextraction and gas chromatography-olfactometry. Journal of Agricultural and Food Chemistry, 59(8), 4062-4070.

Baltes, W., \& Mevissen, L. (1988). Model reactions on roast aroma formation. 6. Volatile reaction-products from the reaction of phenylalanine with glucose during cooking and roasting. Zeitschrift fur Lebensmittel-Untersuchung undForschung, 187(3), 209-214.

Beck, J., Ledl, F., Sengl, M., \& Severin, T. (1990). Formation of acids, lactones and esters through the Maillard reaction. Zeitschrift fur Lebensmittel-Untersuchung und-Forschung, 190, 212-216.

Bianchi, F., Careri, M., Mangia, A., \& Musci, M. (2007). Retention indices in the analysis of food aroma volatile compounds in temperature-programmed gas chromatography: database creation and evaluation of precision and robustness. Journal of Separation Science, 30(4), 563-572.

Buttery, R. G., Teranishi, R., Flath, R., \& Ling, L. (1990). Identification of additional tomato paste volatiles. Journal of Agricultural and Food Chemistry, 38, 792-795.

Chin, S.-T., Eyres, G. T., \& Marriott, P. J. (2011). Identification of potent odourants in wine and brewed coffee using gas chromatography-olfactometry and comprehensive two-dimensional gas chromatography. Journal of Chromatography A, 1218(42), 7487-7498.

Cottier, L., Descotes, G., Neyret, C., \& Nigay, H. (1989). Pyrolyse de sucres. Analyse des vapeurs de caramels industriels. Industries Alimentaires et Agricoles, 106(78), 567-570.

Darriet, P., Pons, M., Henry, R., Dumont, O., Findeling, V., Cartolaro, P., et al. (2002). Impact odorants contributing to the fungus type aroma from grape berries contaminated by powdery mildew (Uncinula necator); Incidence of Enzymatic Activities of the Yeast Saccharomyces cerevisiae. Journal of Agricultural and Food Chemistry, 50(11), 3277-3282.

Dorfner, R., Ferge, T., Kettrup, A., Zimmermann, R., \& Yeretzian, C. (2003). Real-time monitoring of 4-vinylguaiacol, guaiacol, and phenol during coffee roasting by resonant laser ionization time-of-flight mass spectrometry. Journal of Agricultural and Food Chemistry, 51(19), 5768-5773.

Engel, W., Bahr, W., \& Schieberle, P. (1999). Solvent assisted flavour evaporation: a new and versatile technique for the careful and direct isolation of aroma compounds from complex food matrices. European Food Research and Technology, 209(3), 237-241.

Fagerson, I. S. (1969). Thermal degradation of carbohydrates. A review. Journal of Agricultural and Food Chemistry, 17(4), 747-750.

Feron, G., Bonnarme, P., \& Durand, A. (1996). Prospects for the microbial production of food flavours. Trends in Food Science E' Technology, 7(9), 285-293.

Goretti, G., Liberti, A., \& Dipalo, C. (1980). Gas-chromatographic investigation on caramel aroma. Annali di Chimica, 70(5-6), 277-284.

Guichard, E., Kustermann, A., \& Mosandl, A. (1990). Chiral flavour compounds from apricots. Distribution of gamma-lactone enantiomers and stereodifferentiation of dihydroactinidiolide using multi-dimensional gas chromatography. Journal of Chromatography, 498(2), 396-401.

Ito, H. (1977). The formation of maltol and isomaltol through degradation of sucrose. Agricultural and biological chemistry, 41(7), 1307-1308.

Limacher, A., Kerler, J., Davidek, T. Schmalzried, F., \& Blank, I. (2008). Formation of furan and methylfuran by Maillard-type reactions in model systems and food. Journal of Agricultural and Food Chemistry, 56(10), 3639-3647.

Mac Namara, K., Howell, J., Huang, Y. L., \& Robbat, A. (2007). Analysis of gin essential oil mixtures by multidimensional and one-dimensional gas chromatography/ mass spectrometry with spectral deconvolution. Journal of Chromatography A, 1164(1-2), 281-290. 
Martin, B., Etiévant, P., \& Le Quéré, J. L. (1991). More clues of the occurrence and flavor impact of solerone in wine. Journal of Agricultural and Food Chemistry, 39(8), 1501-1503.

Miranda-Lopez, R., Libbey, L. M., Watson, B. T., \& McDaniel, M. R. (1992). Odor analysis of pinot noir wines from grapes of different maturities by a gas chromatography-olfactometry technique (osme). Journal of Food Science, 57(4), 985-993. and 1019.

Miyazato, H., Nakamura, M., Hashimoto, S., \& Hayashi, S. (2013). Identification of the odour-active cyclic diketone cis-2,6-dimethyl-1,4-cyclohexanedione in roasted Arabica coffee brew. Food Chemistry, 138(4), 2346-2355.

Miyazawa, T., Gallagher, M., Preti, G., \& Wise, P. M. (2008). Synergistic mixture interactions in detection of perithreshold odors by humans. Chemical Senses, 33(4), 363-369.

Naef, R., Jaquier, A., Boschung, A. F., \& Lindstroem, M. (1995). The sherry-lactones and solerone. Their identification in dried figs. Flavour and Fragrance Journal, 10(4), 243-247.

Paravisini, L., Gourrat-Pernin, K., Gouttefangeas, C., Moretton, C., Nigay, H., Dacremont, C., et al. (2012). Identification of compounds responsible for the odorant properties of aromatic caramel. Flavour and Fragrance Journal, 27(6), 424-432.

Pittet, A. O., Rittersbacher, P., \& Muralidhara, R. (1970). Flavor properties of compounds related to maltol and isomaltol. Journal of Agricultural and Food Chemistry, 18(5), 929-933.

Pons, I., Garrault, C., Jaubert, J. N., Morel, J., \& Fenyo, J. C. (1991). Analysis of aromatic caramel. Food Chemistry, 39(3), 311-320.
Pons, I., Jaubert, J., Morel, J., \& Fenyo, J. (1995). GC and sensory techniques coupled in caramel flavor analysis. Perfumer E' Flavorist, 20(2), 15-18.

Sanz, C., Maetztu, L., Zapelena, M. J., Bello, J., \& Cid, C. (2002). Profiles of volatile compounds and sensory analysis of three blends of coffee: influence of different proportions of Arabica and Robusta and influence of roasting coffee with sugar. Journal of the Science of Food and Agriculture, 82, 840-847.

Schnermann, P., \& Schieberle, P. (1997). Evaluation of key odorants in milk chocolate and cocoa mass by aroma extract dilution analyses. Journal of Agricultural and Food Chemistry, 45, 867-872.

Seeley, J. V., \& Seeley, S. K. (2013). Multidimensional gas chromatography: fundamental advances and new applications. Analytical Chemistry, 85, 557-578.

Thomsen, M., Martin, C., Mercier, F., Tournayre, P. Berdague, J. L, Thomas-Danguin, T., et al. (2012). Investigating semi-hard cheese aroma: relationship between sensory profiles and gas chromatography-olfactometry data. International Dairy Journal, 26(1), 41-49.

Tranchida, P. Q., Sciarrone, D., Dugo, P., \& Mondello, L. (2012). Heart-cutting multidimensional gas chromatography: a review of recent evolution, applications, and future prospects. Analytica Chimica Acta, 716, 66-75.

Van den Dool, H. \& Kratz, P. D. (1963). A generalization of the retention index system including linear temperature programmed gas-liquid partition chromatography. Journal of Chromatography, 11, 463-471.

Zehentbauer, G., \& Reineccius, G. A. (2002). Determination of key aroma components of Cheddar cheese using dynamic headspace dilution assay. Flavour and Fragrance Journal, 17(4), 300-305. 